\title{
Achieving Very Low Levels of Detection: An Improved Surface-Enhanced Raman Scattering Experiment for the Physical Chemistry Teaching Laboratory
}

\author{
Brian G. McMillan*
}

WestCHEM, Department of Pure and Applied Chemistry, University of Strathclyde, 295

Cathedral Street, Glasgow G1 1XL, Scotland.

\section{ABSTRACT}

This experiment was designed and successfully introduced to complement the nanochemistry taught to undergraduate students in a useful and interesting way.

Colloidal Ag nanoparticles were synthesized by a simple, room-temperature method and the resulting suspension was then used to study the Surface-Enhanced Raman Scattering (SERS) of methylene blue. The colloid was also characterized by UV-Visible spectroscopy and these results were used to help explain some of the observed SERS features. The students looked at the effects of concentration and acquisition time on the measured SERS spectra, and the final part of the experiment was based around using their newly-acquired knowledge to investigate the lowest concentration of methylene blue that could be detected. Concentrations of $5 \times 10^{-10} \mathrm{M}$ were routinely achieved. The combination of UV-Visible spectroscopy, SERS and nanochemistry made for an interesting and thought-provoking laboratory experience.

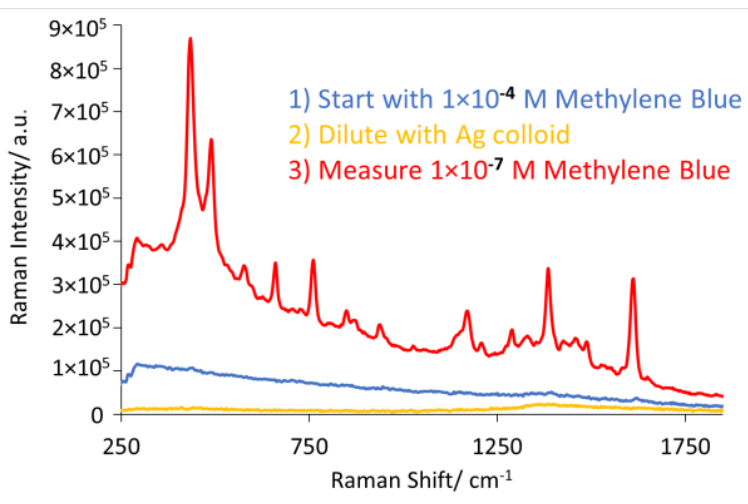




\section{KEYWORDS}

Upper-Division Undergraduate, Analytical Chemistry, Laboratory Instruction, Physical Chemistry, Hands-On Learning/Manipulatives, Problem Solving/Decision Making,

With nanochemistry now a firmly-established subject in undergraduate lecture courses, it is important that this learning is consolidated by practical, laboratory work. If such an experiment can also exploit nanochemistry for a useful purpose, then this will further reinforce its relevance and applicability. This experiment combines a simple, room temperature synthesis of a Ag nanoparticle colloid and Surface-Enhanced Raman Scattering (SERS) to achieve levels of detection of methylene blue (MB) down to $5 \times 10^{-10}$ M. The colloids are also characterised by UV-Visible spectroscopy and this experiment encourages the students to bring together the results of both spectroscopic techniques to help explain what they observe. The students build on their initial results and understanding and then use their newly-acquired knowledge to design a short investigation to establishes their minimum level of detection of MB. While the experiment was developed and taught from a physical chemistry viewpoint, it also has a clear analytical chemistry theme.

SERS exploits the massive enhancement (up to $10^{9}$ times) $)^{1,2}$ of the Raman signal which is observed when a Raman active molecule is adsorbed on a SERS active substrate. Since being first reported in $1974,{ }^{3}$ the technique has been widely applied ${ }^{4}$ and the enhancement mechanism has been studied.5,6 Current theory suggests that that there are two possible mechanisms. ${ }^{4}$ Firstly, an electromagnetic enhancement is caused when a surface plasmon mode of the metallic substrate is excited by the incident light. This amplifies the local electromagnetic field experienced by the adsorbed analyte molecules. ${ }^{7}$ Preferential amplification occurs in gaps formed when nanoparticles 
aggregate together, as localized plasmon modes can couple. ${ }^{8,9}$ These 'hot-spots' of activity have given rise to single molecule detection. ${ }^{10}$ The second mechanism is a chemical one involving charge transfer between the test molecule and the metal;11 the excitation wavelength can resonate with metal-molecule electronic states. ${ }^{12,13}$ The electromagnetic mechanism is thought to be the predominant one ${ }^{8}$ as SERS is still possible when no charge transfer mechanism exists.

The vivid colours of colloidal $\mathrm{Au}, \mathrm{Ag}$ and $\mathrm{Cu}$ metal particles are caused by their surface plasmon modes so these are widely used as SERS substrates. ${ }^{14}$ Silver colloidal nanoparticles are highly active ${ }^{15}$ and silver salts are much less expensive than gold ones, which is an important consideration when designing any teaching experiment.

There are several reported synthetic methods for preparing SERS active colloidal Ag nanoparticles ${ }^{16,17}$ but not all of them are all accessible or practical for teaching laboratory experiments. Some have been applied to SERS experiments, ${ }^{18,19,20}$ but the emphasis this experiment is different. ${ }^{21}$ Unlike these previously-reported experiments, this one focusses on combining a simple, rapid, room-temperature synthesis (based on that developed by Leopold and Lendl) ${ }^{22}$ with UV-visible and SERS spectroscopy to guide the students towards investigating the minimum level of detection of MB. By reducing the time and complexity of the synthesis step, the students can focus on characterizing and using their colloid. In contrast with the other reported SERS teaching experiments, the students then build on what they learn during these characterization steps to design and implement a short investigation which enables the minimum level of detection of MB to be determined; the levels achieved are impressively low for an undergraduate teaching laboratory experiment. Sequentially introducing the analysis method and instrumental parameters allows the students to glean the important parameters as they progress through the work. This experiment begins as a fullydescribed one, but it then progresses to give the students some freedom to investigate 
for themselves. Reducing the synthesis time enables this to be competed in a short laboratory session. Instructors can choose how much additional guidance to give to their students when conducting the investigation section of the experiment, allowing the experiment to be tailored to match students' abilities.

In addition to the synthesis advantages listed above, using MB as the analyte is less hazardous, more convenient and less expensive than other potential test molecules such as pyridine ${ }^{18}$ or Rhodamine $6 \mathrm{G} .{ }^{20}$

\section{CHEMICALS AND EQUIPMENT}

All chemicals were reagent grade and all solutions were prepared using deionised water.

UV-Visible absorbance spectra were recorded using an Ocean Optics USB2000+ Spectrometer, the light source was a tungsten lamp and the cuvette holder was built inhouse. The Raman instrument was a Centice MMS Raman Spectrometer with an excitation wavelength of $784 \mathrm{~nm}$. Both of these instruments were controlled using Ocean Optics SpectraSuite software, which offered an additional advantage to the students as everything was controlled through a single computer interface. This experiment can be adapted and optimized to suit alternative spectroscopic equipment.

\section{COLLOID SYNTHESIS}

The Ag colloid was synthesized as described by Leopold and Lendl ${ }^{22}$ with only slight modification for practical, teaching laboratory purposes. Full details are in the

95 supplementary material but briefly, the students were supplied with stock solutions of $1 \mathrm{mM} \mathrm{AgNO}_{3}$ and $0.3 \mathrm{M} \mathrm{NaOH}$ whereas the hydroxylamine was supplied as a solid as solutions of it were found to be unstable, lasting no more than a few days. The students prepared the reducing solution and added this to the $\mathrm{AgNO}_{3}$ (with stirring) in an Erlenmeyer Flask. The colloid goes through a series of color changes before finally becoming milky-yellow; it is ready for use in 2-3 minutes. The advantages of using this 
process in a teaching experiment are that it can be carried out at room temperature and useable colloid is rapidly prepared - the students know quickly by the color change if their synthesis has been successful and can readily produce another batch if necessary. Other syntheses, in contrast, generally take significant time ${ }^{18}$ and require heating ${ }^{16}$ or cooling ${ }^{17}$ of the reagents.

\section{TEST REAGENT}

Methylene blue (MB) was chosen as the test reagent as it is both inexpensive and gives reproducible SERS spectra showing many vibrational bands. The normal Raman (non-SERS) spectrum of even the $1 \times 10^{-4} \mathrm{M}$ MB stock solution supplied to the students showed very few features.

\section{UV-VISIBLE CHARACTERIZATION}

The absorbance spectrum of the diluted colloid ( $c$. 5-fold dilution - see supplemental material for details) was initially measured and the typical absorbance profile of Ag nanoparticles was observed (Figure 1).

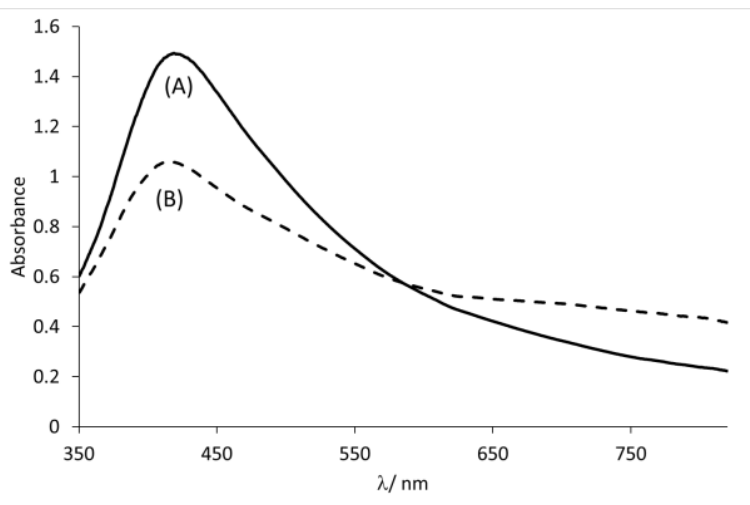

Figure 1. UV-Visible spectra of Ag colloid without (A) and with (B) added $\mathrm{NaCl}$ solution.

The broad absorption band corresponds to the surface plasmon resonance of the suspended Ag nanoparticles, which is caused by a collective, quantized oscillation of the free electrons in the metal nanoparticles. The resonance is initiated by the electric field of the incident photons. One drop of $2 \mathrm{M} \mathrm{NaCl}$ solution was then added to the cuvette 
and the spectrum was re-measured after $60 \mathrm{~s}$. The changes in the absorbance are caused by electrostatic aggregation of the colloidal particles ${ }^{22,14}$ with $\mathrm{Cl}^{-}$ions bridging between particles and these changes are used to help explain some of the SERS features recorded during the next part of the experiment. It is noted in passing that the SpectraSuite software continuously acquires and displays the absorption spectra as the aggregation proceeds, allowing the students to watch the changes in real time.

\section{SURFACE-ENHANCED RAMAN SPECTROSCOPY}

Initial Measurements and Concentration Dependence

For comparison, the initial Raman spectra of the Ag colloid and the $1 \times 10^{-4} \mathrm{M} \mathrm{MB}$ stock solution were measured and neither of these showed significant Raman scattering (Figure 2). The SERS spectrum of the colloid with MB $\left(1 \times 10^{-7} \mathrm{M}\right)$ was then measured and a large increase in the signal was observed. This is also shown in Figure 2.

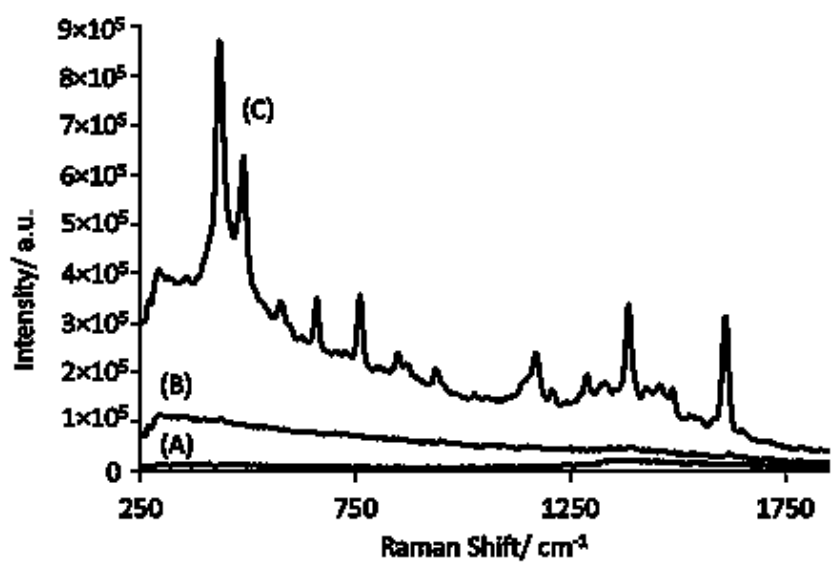

Figure 2. Raman spectra of the Ag colloid (A), $1 \times 10^{-4} \mathrm{M} \mathrm{MB}$ solution (B) and SERS of the Ag colloid with $1 \times 10^{-7} \mathrm{M} \mathrm{MB}(\mathrm{C})$.

The SERS intensity as a function of MB concentration was then measured to give the plot in Figure 3, illustrating the linearity of the SERS technique in this concentration range. 


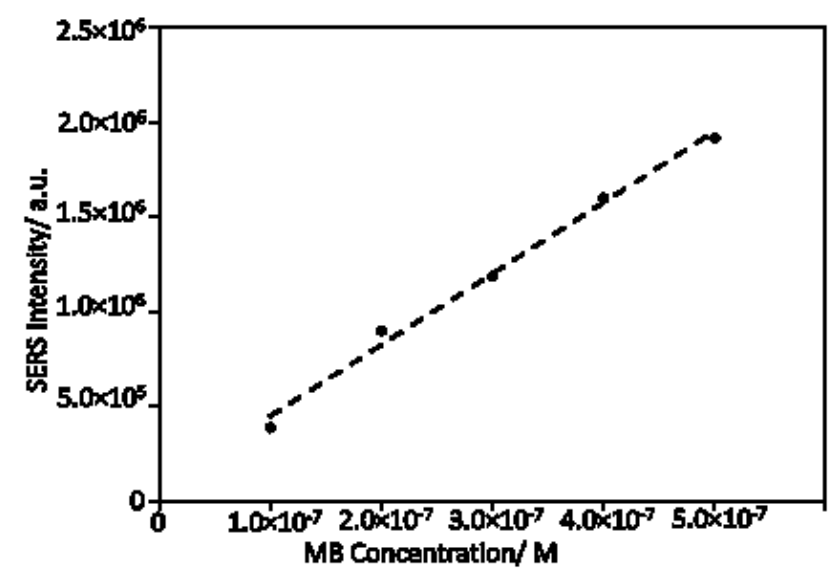

Figure 3. Plot of SERS intensity vs. MB concentration.

For the next measurement, one drop of $2 \mathrm{M} \mathrm{NaCl}$ was added to the sample vial containing the final MB concentration and the SERS spectrum was re-measured after $60 \mathrm{~s}$. A further, large enhancement of the signal was observed as shown in Figure 4. A $\mathrm{NaCl}$ concentration in the $0.1 \mathrm{M}-0.2 \mathrm{M}$ range was found to work well in this experiment.

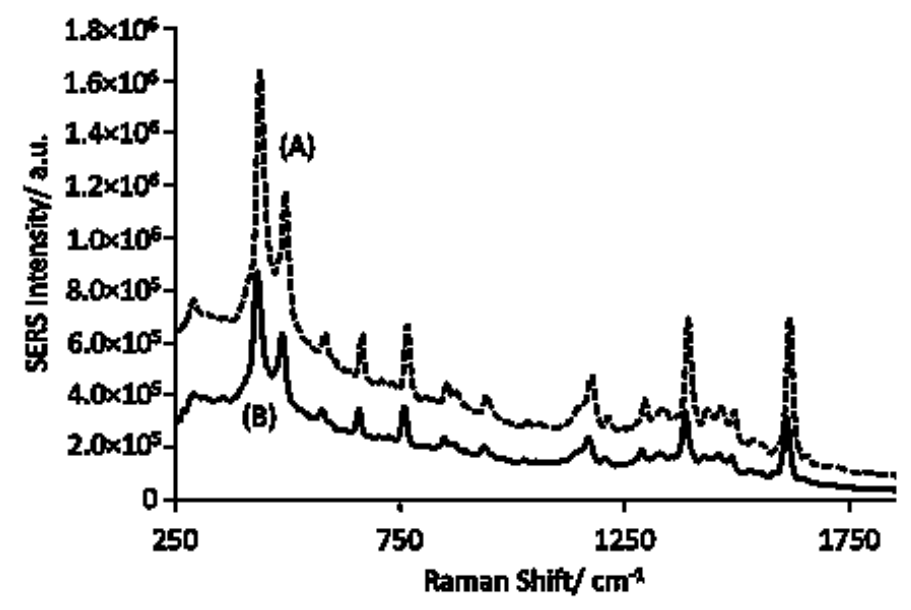

Figure 4. SERS spectra of $\mathrm{MB}$ adsorbed onto a $\mathrm{Ag}$ colloid with $(\mathrm{A})$ and without $(\mathrm{B})$ added $\mathrm{NaCl}$ solution.

Adding $\mathrm{NaCl}$ causes the nanoparticles to aggregate, ${ }^{22}$ which increases the overall scattering intensity as the effective particle size increases. The aggregation is also 
thought to increase the number of 'hot-spots', 8 which are formed when plasmon modes of nanoparticles brought together by aggregation couple; preferential electromagnetic amplification occurs here. A higher measured 'absorbance' (there will be light lost due to scattering from the larger particles so extinction is more correct) in the UV-Visible spectrum at the laser wavelength of $784 \mathrm{~nm}$ was observed when $\mathrm{NaCl}$ was added. This can therefore be used, in simple terms, to illustrate why the SERS intensity increases. There are more possible interaction events between the nanoparticles and the excitation light. When synthesised, the colloidal particles are capped and protected from aggregation by the hydroxylamine as this leaves them with a surface charge. ${ }^{14}$ The addition of ions to the suspension screens this charge and allows aggregation. The other potential effects of the chloride ion on the SERS signal (through the charge transfer mechanism) were considered too advanced for this experiment ${ }^{14}$ but this could be further explored and developed. In any case, this illustrates that the SERS intensity also depends on the shape/morphology of the nanostructure.

Spectrometer Acquisition Time Dependence

The dependence of the SERS signal on the instrument's acquisition time was then measured using a constant MB concentration. The data are plotted in Figure 5.

This part of the experiment was designed to show that the measured Raman scattering intensity could also be improved (up to a point) by changing some of the instrumental parameters. The initial linear increase in intensity cannot be maintained as the acquisition time continues to increase; the students are asked to comment on this in their laboratory reports. This section of work was specifically included to assist with the final stage of the experiment. 


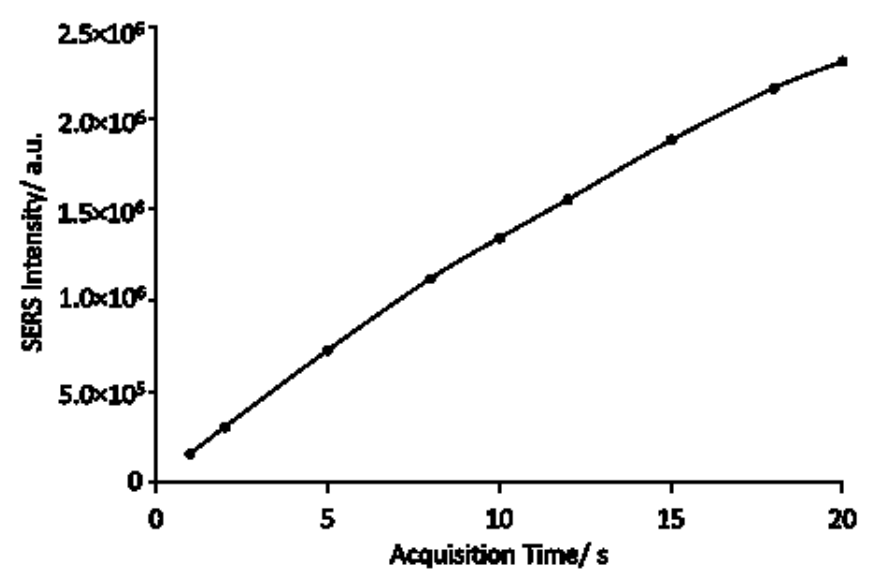

Figure 5. Plot of SERS Intensity vs. Spectrometer Acquisition Time for $4 \times 10^{-7} \mathrm{M}$ MB solution.

Investigation of the Minimum Level of Detection

Here, the students were asked to bring the previous work together to determine the minimum concentration of MB that they could detect using the SERS technique. Basic instructions were given to them (see supplemental information) and the students were asked to decide at what concentration they were sure they were first detecting MB by noting when the main peak at $c a .430 \mathrm{~cm}^{-1}$ first appeared. This fine qualitative judgement formed part of the assessment of the experiment. Concentrations of MB down to $5 \times 10^{-10} \mathrm{M}$ were detected and example spectra are shown in Figure 6 . These data were recorded with a $20 \mathrm{~s}$ acquisition time and show Raman spectra form the colloid itself, together with the first evidence of MB detection at $5 \times 10^{-10} \mathrm{M}$ where $1 \mu \mathrm{l}$ of a solution containing $1 \times 10^{-6} \mathrm{M} \mathrm{MB}$ was added to $2 \mathrm{~cm}^{3}$ of colloid. To confirm that this really was MB, a further $1 \mu \mathrm{l}$ aliquot was added and the increased Raman activity in the region around $450 \mathrm{~cm}^{-1}$ was taken as confirmation. Further dilution of the MB and the addition of $\mathrm{NaCl}$ may have yielded a lower detection threshold, but the recorded spectra were sufficiently good for the purpose of the experiment. 


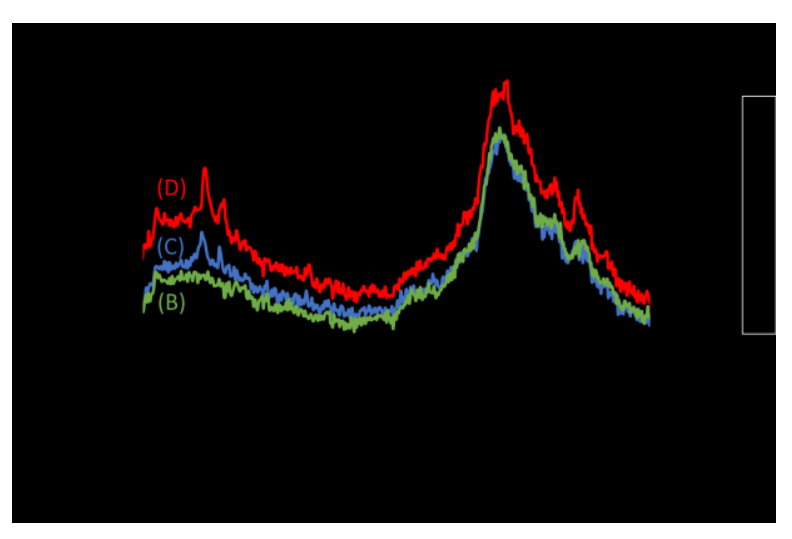

Figure 6. Determining the minimum level of MB that can be detected. Spectrum $(A)$ is the same as Spectrum $(C)$ from Figure 2, which is Ag colloid with $1 \times 10^{-7} \mathrm{M} \mathrm{MB}$. This is included to show the peaks developing. Spectrum $(B)$ is the colloid alone, $(C)$ contained $5 \times 10^{-10} \mathrm{M} \mathrm{MB}$ and (D) $1 \times 10^{-9} \mathrm{M} \mathrm{MB}$. Acquisition time was fixed at $20 \mathrm{~s}$ and no $\mathrm{NaCl}$ was added.

\section{HAZARDS AND PRECAUTIONS}

The prepared solutions provided for the students are at low concentration so standard chemical handling precautions, including laboratory coats, safety glasses and gloves are appropriate; no special precautions are necessary. Hydroxylamine hydrochloride is used as a solid and is considered harmful and irritant in this form. Gloves must be worn when weighing out this material. Alternatively, this can be given to the students pre-weighed in a vial or as a freshly-prepared solution (see supplemental information for details) for use; the required solution concentration is very low.

The Raman spectrometer used in this experiment has a safety interlock which prevents exposure to laser radiation when the cell compartment is open. Other spectrometers, particular Raman microscope equipment, might require additional precautions.

\section{DISCUSSION OF IMPACT}

During the course of the academic year over 130 third-year undergraduate students carried out the experiment in the physical chemistry teaching laboratory. The students worked in pairs and took between two and three hours to work through the procedure. 
Each student was required to submit a laboratory report which included answering 'quiz questions' based on their results. A cross-section of the class was also interviewed on completion of the experiment to gauge their interest and understanding.

Examination of the marked reports showed that on the whole, the students engaged with the experiment with the class average mark being $81 \%$. The students' answers to the 'quiz questions' showed that their understanding of Raman Spectroscopy in general and SERS in particular was enhanced by this practical activity, and the linking of the absorbance and Raman spectra proved to be thought-provoking and encouraged much discussion and interest. The analytical as well as the physical and nanochemistry aspects were also highlighted by the questions asked of the students.

During the course of the year, a few issues came to light. The most common 230 problem encountered was with consistently mixing very small volumes (e.g. 1-2 $\mu$ l) of liquid into larger (e.g. $2 \mathrm{~cm}^{3}$ ) ones. To counter this, a practical demonstration was given and the experimental procedure has been updated to stress the need for careful and consistent mixing. This problem caused non-linearity in the types of plot shown in Figure 3.

The students were particularly interested and somewhat surprised at the low MB detection limit achieved using the SERS effect, particularly given the virtual non-activity of MB in normal Raman. The stark contrast between the lack of Raman activity from the MB stock solution and the detailed spectrum recorded when the first $2 \mu \mathrm{l}$ of $\mathrm{MB}$ were added to the colloid really caught the students' attention. The students also were also interested in using Raman spectroscopy in general as while they do learn about the technique, they had never used it before. They were much more accustomed to using FTIR spectroscopy and the fact that Raman spectra can be collected in aqueous solution was also of interest. 
The investigation part of the experiment can be modified to suit the abilities of the students, with more or less help or instruction given on how to complete the task. What is included in the supplemental information is what was considered appropriate for the class at the time. It is possible to achieve a lower level of MB detection by using a more highly diluted MB stock solution ( $1 \mu 1$ was the lowest volume that could be pipetted in the lab) and adding $\mathrm{NaCl}$ would further enhances the signal; $1 \times 10^{-11} \mathrm{M}$ was achieved during development. This would, however, add considerable time to the experiment and could change its emphasis too much. It is also noted that the absolute level of detection achievable will be dependent on the available instrumentation.

In conclusion, this experiment was successfully implemented and it did reinforce the lecture classes on nanochemistry, absorbance spectroscopy and Raman spectroscopy by introducing a relevant practical activity. The investigation part of the experiment encouraged to students to think more independently about what they were doing and gave them some experience of designing and implementing a short experimental protocol which required skill and judgement. Minimum MB levels in the $1 \times 10^{-8} \mathrm{M}$ to $5 \times 10^{-10} \mathrm{M}$ range were achieved by all of the students.

\section{ASSOCIATED CONTENT}

Supporting Information

The full laboratory manual, staff guidelines and marking scheme are provided.

\section{AUTHOR INFORMATION}

Corresponding Author

265 *E-mail: brian.mcmillan@strath.ac.uk

\section{ACKNOWLEDGMENTS}

Sincere thanks are due to the students, technical staff and postgraduate demonstrators of the Physical and Applied Chemistry Teaching laboratory. 
1. Herrera, G. M.; Padilla, A. C.; Hernandez-Rivera, S. P. Surface Enhanced Raman Scattering (SERS) Studies of Gold. Nanomaterials. 2013, 3, 158-172.

2. Le Ru. E. C.; Etchegoin, P. G. Quantifying SERS enhancements. MRS Bulletin. 2013, 38, 631-640.

3. Fleischmann, M.; Hendra, P. J.; McQuillan, A. J. Raman spectra of pyridine adsorbed at a silver electrode. Chem. Phys. Lett. 1974, 26 (2), 163-166.

4. Sharma, B.; Frontiera, R. R.; Henry, A.-I.; Ringe, E.; Van Duyne, R. P. SERS: Materials, applications, and the future. Materialstoday. 2012, 15 (1-2), 16-25.

5. Jeanmaire. D. L.; Van Duyne, R. P. Surface Raman spectroelectrochemistry. Part I. Heterocyclic, aromatic, and aliphatic amines adsorbed on the anodized silver electrode. J. Electroanal. Chem. Interface Electrochem. 1977, 84 (1), 1-20.

6. Albrecht, M. G.; Creighton, J. A. Anomalously intense Raman spectra of pyridine at a silver electrode. J. Am. Chem. Soc. 1977, 99 (15), 5215-5217.

7. Stiles, P. L.; Dieringer, J. A.; Shah, N. C.; Van Duyne, R. P. Surface-Enhanced Raman Spectroscopy. Annual Rev. Anal. Chem. 2008, 1, 601-626.

8. Maher, R. C. SERS Hot Spots. In Raman Spectroscopy for Nanomaterials Characterization, Kumar C. S. R., Ed.; Springer: Berlin, Germany, 2012; 215260.

9. Shioharaa, A.; Wanga, Y.; Liz-Marzan, L. M. Recent approaches toward creation of hot spots for SERS detection. J. Photochem. Photobiol C: Photochem. Rev. 2014, 21, 2-25.

10. Nie, S.; Emroy, S. R. Probing single molecules and single nanoparticles by surface-enhanced Raman scattering. Science. 1997, 275 (5303), 1102-1105.

11. John R. Lombardi, J. R.; Birke, R. L.; Lu, T.; Xu, J. Charge-transfer theory of surface enhanced Raman spectroscopy: Herzberg-Teller contributions. J. Chem. Phys. 1984, 84, 4174-4180.

12. Jensen, L.; Aikens, C. M.; Schatz, G. C. Electronic structure methods for studying surface-enhanced Raman scattering. Chem. Soc. Rev. 2008, 37, 10611073.

13. Morton, S. M.; Jensen, L. Understanding the Molecule-Surface Chemical Coupling in SERS. J. Am. Chem. Soc. 2009, 131 (11), 4090-4098.

14. Le Ru, E. C.; Etchegoin, P. G. Principles of Surface-Enhanced Raman Spectroscopy; Elsevier: Amsterdam, Netherlands, 2009; 367-375.

15. Eom, S. Y.; Ryu, S. L.; Kim, H. L.; Kwon, C. H. Systematic preparation of colloidal silver nanoparticles for effective SERS substrates. Colloids and Surfaces A: Physicochem. Eng. Aspects. 2013, 422, 39-43.

16. Lee, P. C.; Meisel, D. Adsorption and surface-enhanced Raman of dyes on silver and gold sols. J. Phys. Chem. 1982, 86 (17), 3391-3395.

17. Creighton, J. A.; Blatchford, C. G.; Albrecht, M. G. Plasma resonance enhancement of Raman scattering by pyridine adsorbed on silver or gold sol particles of size comparable to the excitation wavelength. J. Chem. Soc. Faraday Trans. 2. 1979, 75, 790-798.

18. Weaver, J. C.; Norrod, K. Surface-Enhanced Raman Spectroscopy - A Novel Physical Chemistry Experiment for the Undergraduate Laboratory. J. Chem. Educ. 1998, 75, 621-624.

19. Mayhew, H. E.; Frano, K. A.; Svoboda, S. A.; Wustholz, K. L. Using Raman Spectroscopy and Surface-Enhanced Raman Scattering To Identify Colorants in Art: An Experiment for an Upper-Division Chemistry Laboratory. J. Chem. Educ. 2015, 92, 148-152. 
20. Pavel, I. E.; Alnajjar, K. S.; Monahan, J. L.; Stahler, A.; Hunter, H. E.; Weaver, K. M.; Baker, J. D.; Meyerhoefer, A. J.; Dolson, D. A. Estimating the Analytical ans Surface Enhancement Factors in Surface-Enhanced Raman Scattering (SERS): A Novel Physical Chemistry and Nanotechnology Experiment. J. Chem. Educ. 2012, 89, 286-290.

21. Seney, C. S.; Yelverton, J. C.; Eanes, S.; Patel, V.; Riggs, J.; Wright, S.; Bright, R. M. Use of Surface-Enhanced Raman Spectroscopy in Inorganic Syntheses for an Upper-Level Exploratory Lab. J. Chem. Educ. 2007, 84, 132-135.

22. Leopold, N.; Bernhard Lend1, B. A New Method for Fast Preparation of Highly Surface-Enhanced Raman Scattering (SERS) Active Silver Colloids at Room

Temperature by Reduction of Silver Nitrate with Hydroxylamine Hydrochloride. J. Phys. Chem. B. 2003, 107, 5723-5727. 


\section{Demonstrators' Notes}

Preliminary:

- Ensure that the PC, Raman and UV-Vis spectrometer are on with the black cuvette in the cell holder to block the beam.

- Ensure that the stock solution bottles $\left(1 \mathrm{mM} \mathrm{AgNO}_{3}, 0.3 \mathrm{M} \mathrm{NaOH}, 1 \times 10^{-4} \mathrm{M}\right.$ methylene blue and $2 \mathrm{M} \mathrm{NaCl}$ ) are topped up and that there are sufficient micropipette tips, sample vials and hydroxylamine hydrochloride. There is spare colloid in the cupboard.

- There should also be: $1 \times 100 \mathrm{ml}$ Conical Flask, $2 \times 100 \mathrm{ml}$ volumetric flasks, $1 \times 50 \mathrm{ml}$ pipette, $1 \times 10 \mathrm{ml}$ pipette, $1 \times 5 \mathrm{ml}$ pipette, $1 \times 1 \mathrm{ml}$ pipette, $1 \times 1000 \mu$ micropipette, $1 \times 20$ $\mu$ micropipette, 1 glass weighing boat, the marked cuvette and some beakers.

Points to be note:

- Ensure that gloves are being worn.

- Try to get to the students after they have made their colloid and make sure it looks correct.

- Remind them to take two samples of their colloid at the start so that they have a 'clean' one for Part C, explaining that methylene blue contamination is common and that the experiment is sensitive to this.

- The students tend not to mix the solutions enough when adding methylene blue to the colloid - highlight this and demonstrate if needed. Recommend at least 10 inversions of the vial per mixture.

- Try to find the opportunity talk the students through what they are seeing after they measure the Raman spectrum of the colloid + methylene blue. Highlight the lack of peaks in the colloid, methylene blue stock and the intense spectrum with the very dilute methylene blue and the colloid.

- For Part C, ask the students hat their plan is for achieving the minimum level of detection and be prepared to guide them. As a guide, a few $\mu$ l of $1 \times 10^{-6} \mathrm{M}$ methylene blue in $2 \mathrm{ml}$ colloid is required. If their blank shows methylene blue peaks check that they are using the clean colloid sample taken at the start. If they are, give them a vial of the spare colloid to use.

- The Raman peak assignments are on display on the wall should anyone ask! 


\section{Experiment 3C Marking Scheme (25 marks)}

- An Excel spreadsheet can be used to calculate the answers quickly.

- Remember that Excel calculates to many decimal places, so answers may vary slightly.

- If a student miscalculates one part of a question or simply uses the wrong value, remember that they may have calculated the rest correctly using this wrong value e.g. density = mass/volume and if they use the wrong volume then the density will be wrong. In such cases please do the following:

1. Mark the initial mistake as wrong and deduct the mark(s) associated with it.

2. Manually input their incorrect answer into the marking spreadsheet and proceed to mark with these answers (pointing out to student that you have taken their error into account).

3. If any further calculation mistakes are made, do not update spreadsheet answers. Mark them wrong and deduct marks associated. The students only get one 'free' mistake!

4. This method also applies to error calculations.

5. Above all, please use your discretion and seek advice if necessary.

- Do not use negative marks, once a student has got zero for one part of a question move on to the next part.

\section{Report Quality}

If untidy, poorly explained, poorly structured, no aims etc. deduct 1.

\section{$\underline{\text { Results/Calculations }}$}

If there are poor practical results, results not clearly presented e.g. data not tabulated, no example of each calculation, unclear calculations etc. deduct as appropriate.

Q1 - The students are asked to plot their two UV-VIS spectra on the same chart and to rescale the $x$ axis to range from $350-820 \mathrm{~nm}$. The spectra should be labelled to show which has the $\mathrm{NaCl}$ added. Typical spectra are shown in Fig. 1 below.

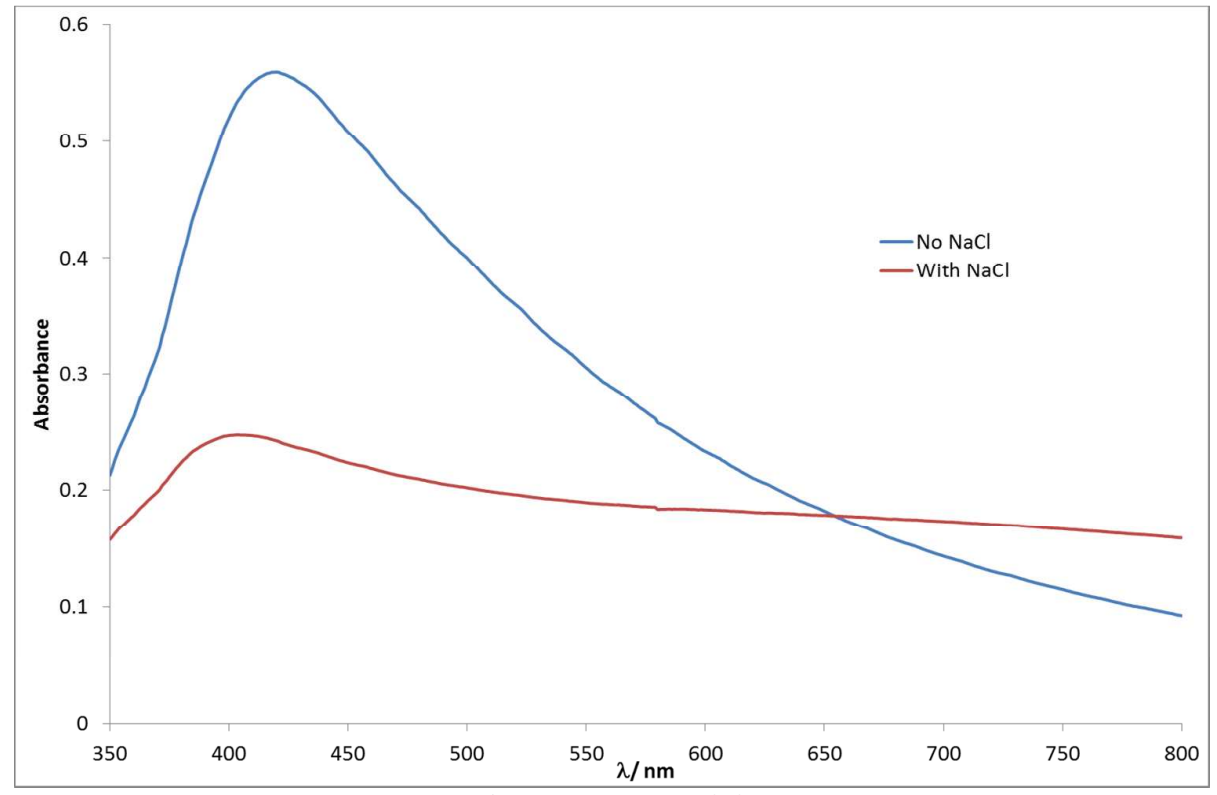

Figure 1-UV-VIS Spectra

(2 marks for correctly labelled plot) 
Q2 - This question asks the students to plot their Raman spectra for the Ag colloid and the methylene blue (MB) stock solutions and to highlight some Raman bands in the MB spectrum. Typical data in Fig. 2 below.

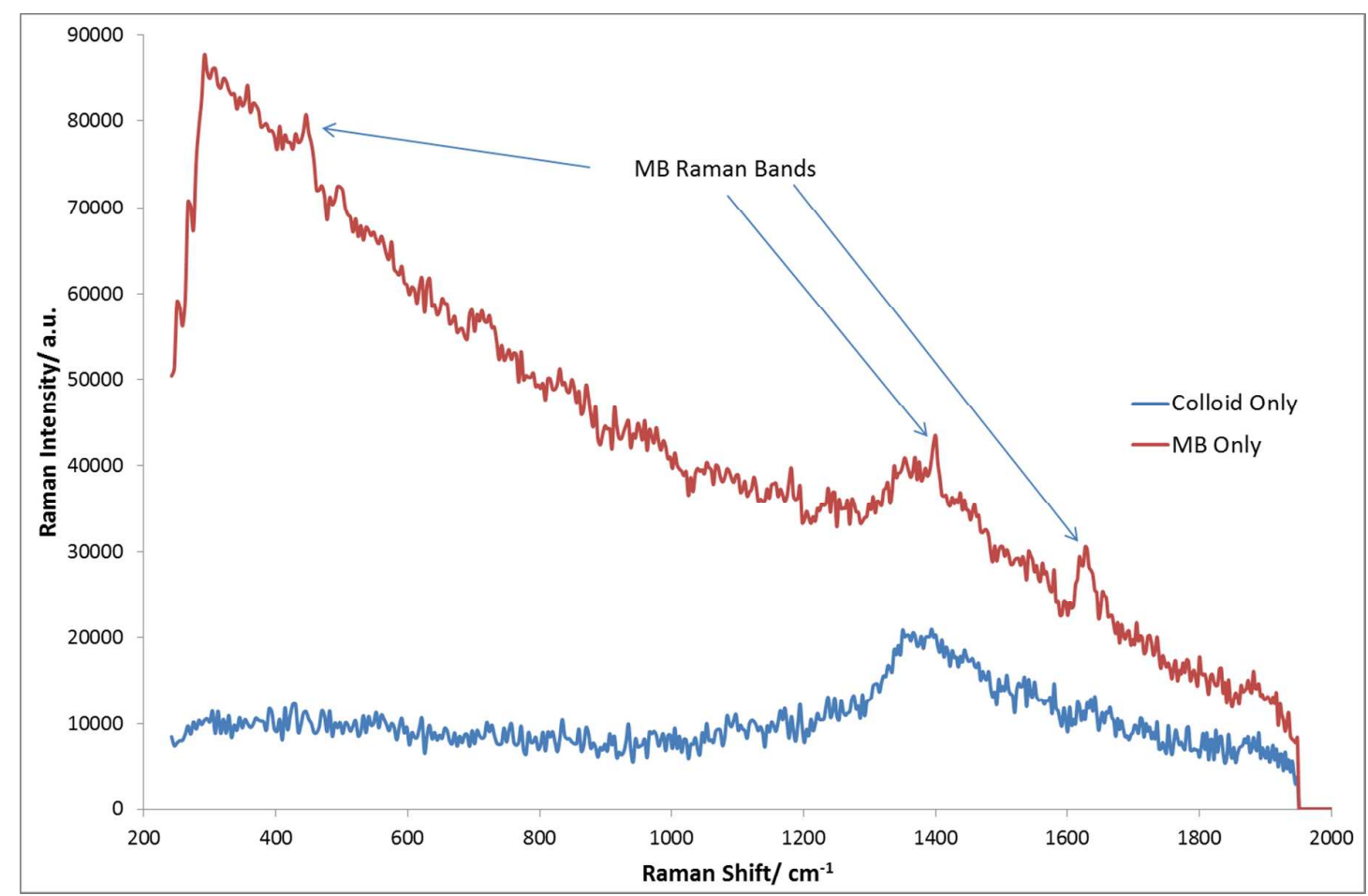

Figure 2-Raman spectra of Ag colloid and MB stock solution

Please note that sometimes, the large fluorescence background in the MB spectrum is sometimes absent and that the Raman bands are not particularly obvious - credit reasonable attempts to identify peaks.

\section{( 2 marks for correctly labelled plot, 1 mark for labelling some peaks)}

Q3 - The students are asked to plot the MB stock solution spectrum, their Ag Colloid/10 $\mu \mathrm{MB}$ spectrum and their $\mathrm{Ag}$ Colloid/10 $\mu \mathrm{lMB} / \mathrm{NaCl}$ spectrum. Typical spectra are shown in Fig. 3 below. The students are asked to comment on the differences between the spectra.

The key features are that the combined colloid/MB spectrum shows a great surface enhanced Raman (SERS) effect caused by the adsorption of the MB molecules onto the surface of the $\mathrm{Ag}$ nanoparticles. Virtually nothing is observed in the 'normal' Raman spectrum of the MB stock solution but the SERS spectrum contains many features, even at the much reduced concentration used.

The addition of $\mathrm{NaCl}$ causes the SERS signal intensity to increase further. 


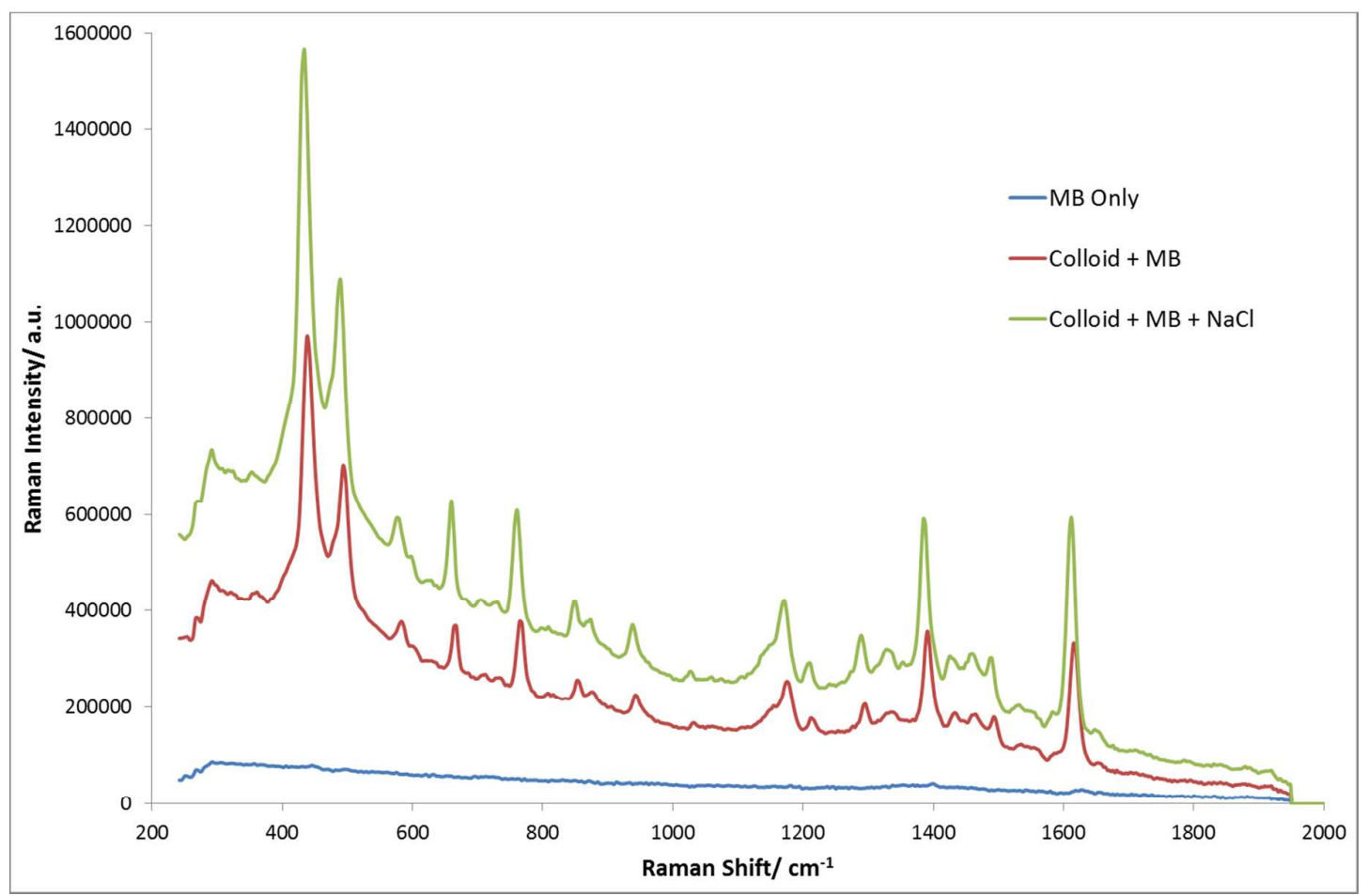

Figure 3 -SERS Spectra

\section{( 2 marks for correctly labelled plot, 1 mark for discussion of features)}

Q4 - The students are asked to explain why the signal intensity increases on the addition of $\mathrm{NaCl}$. The students are asked to make reference to their UV-VIS data and the laser wavelength used, which was $784 \mathrm{~nm}$. The explanation is that the $\mathrm{NaCl}$ causes aggregation of the nanoparticles. The addition of the $\mathrm{NaCl}$ causes a general overall reduction and broadening of the UV-VIS absorption feature, but the absorption at the laser wavelength is actually higher when $\mathrm{NaCl}$ is added. This indicates that the number of particles interacting with the laser wavelength is higher in the aggregated colloid and thus the SERS enhancement is greater. The students are given all of this information in the script, but they will have to piece it together to get to the answer!

\section{(2 marks for the explanation)}

Q5 - The Students are asked to include their tabulated data as below:

\begin{tabular}{|c|c|c|}
\hline MB Volume $/ \mu \mathrm{l}$ & MB Molarity/ mol dm & Raman Intensity/ a.u. \\
\hline 2 & $1.00 \mathrm{E}-07$ & 190635 \\
\hline 4 & $2.00 \mathrm{E}-07$ & 335023 \\
\hline 6 & $3.00 \mathrm{E}-07$ & 445216 \\
\hline 8 & $4.00 \mathrm{E}-07$ & 633892 \\
\hline 10 & $5.00 \mathrm{E}-07$ & 820034 \\
\hline
\end{tabular}

Table 1 - Raman Intensity and concentration

They are asked to plot and comment on the data. The plot is shown in Fig. 4, it should be a straight line. 


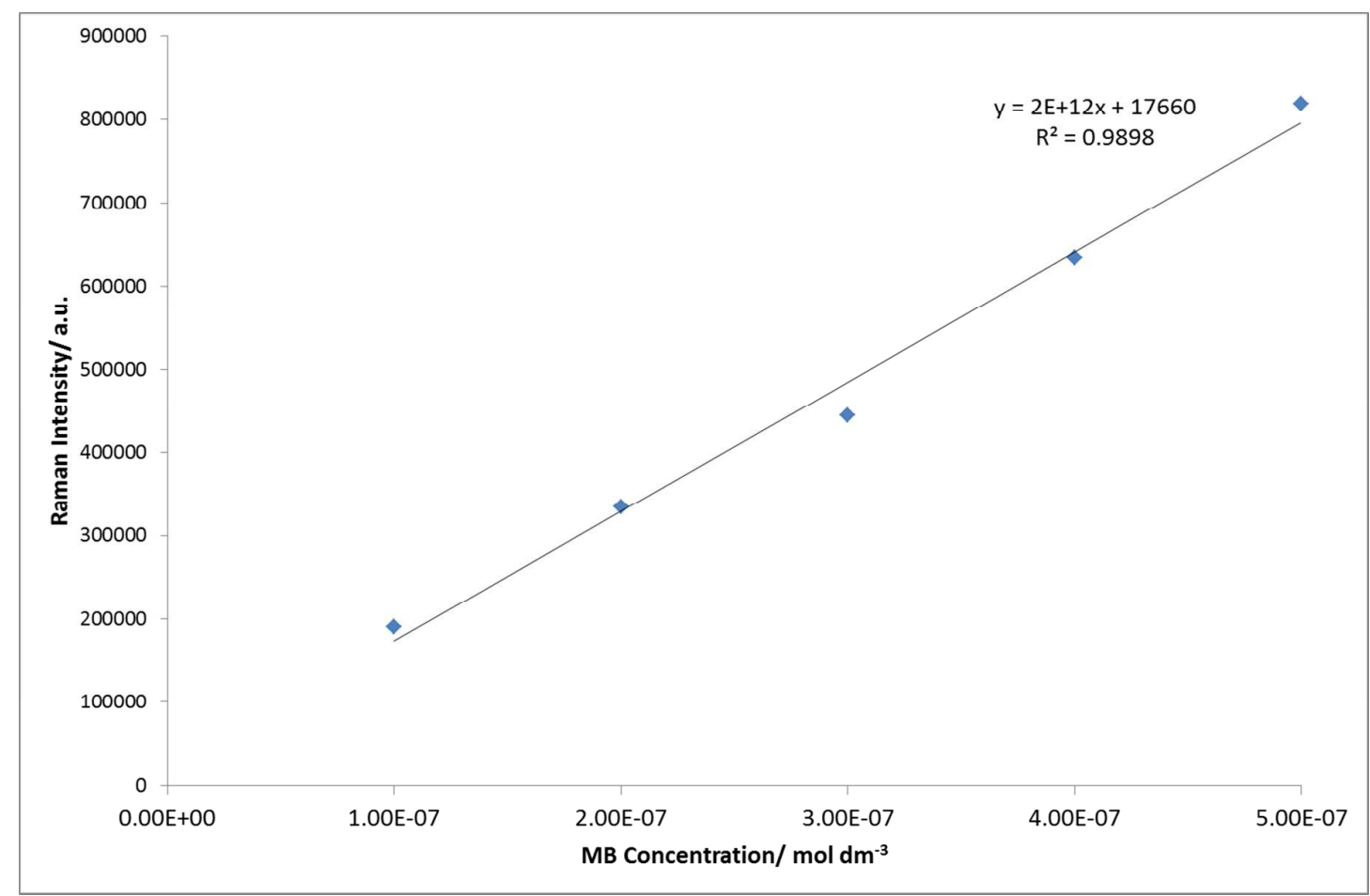

Figure 4 - Raman Intensity vs. Concentration.

(1 mark for table, 2 marks for plot, 1 mark for comment)

Q6 - The students are asked to include their tabulated data as below:

\begin{tabular}{|c|c|}
\hline Integration Time/s & Raman Intensity/ a.u. \\
\hline 1 & 78113 \\
\hline 2 & 148778 \\
\hline 5 & 372879 \\
\hline 8 & 577631 \\
\hline 10 & 720117 \\
\hline 12 & 821703 \\
\hline 15 & 986437 \\
\hline 18 & 1126670 \\
\hline 20 & 1192454 \\
\hline
\end{tabular}

Table 2 - Raman Intensity and integration time

They are asked to plot these data (Fig. 5) and comment on the plot. The plot should initially be linear, but should flatten out at the higher integration times. 


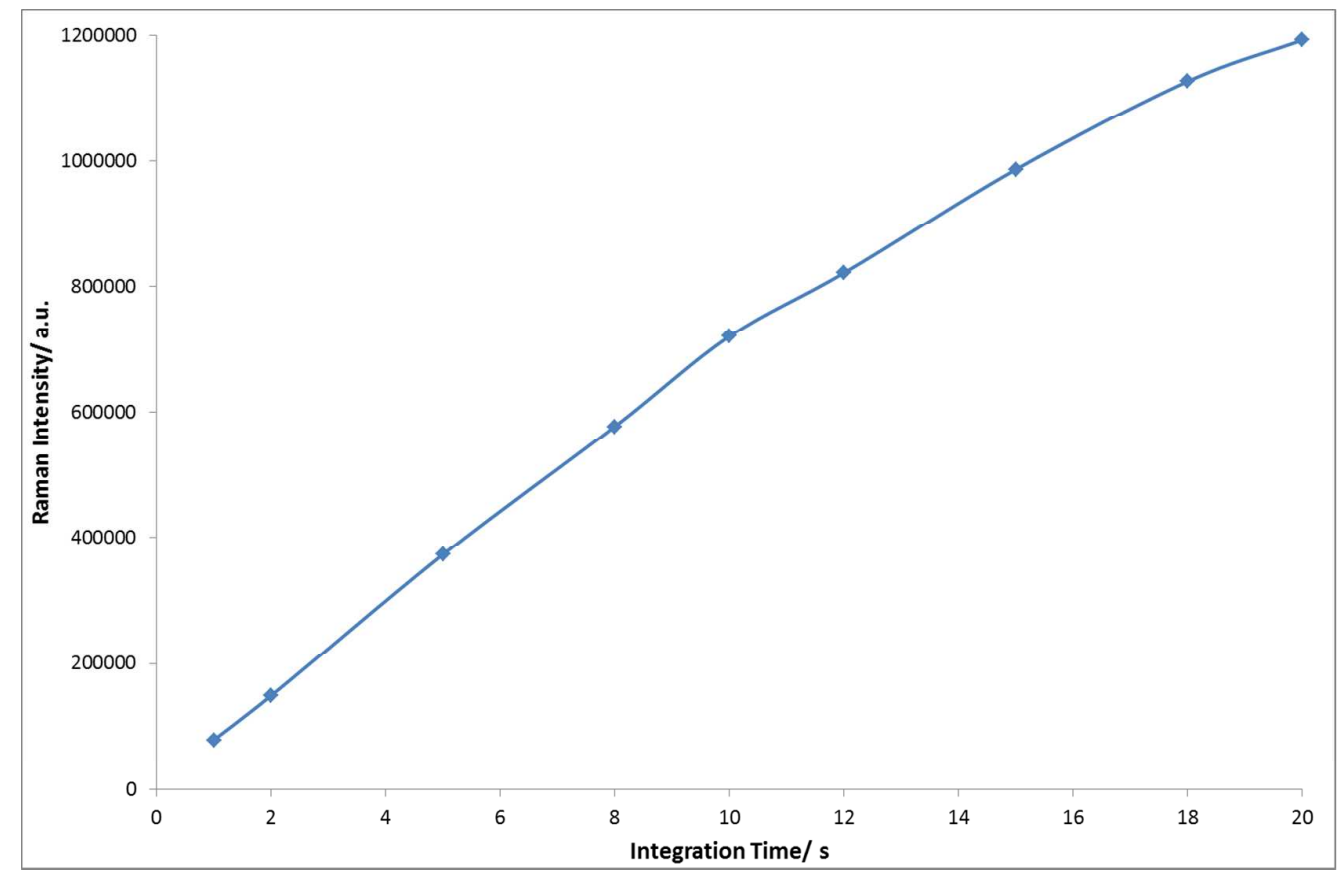

Figure 5-Raman Intensity vs. Integration time

(1 mark for table, 2 marks for plot, 1 mark for comment)

Q7 - The final part of the experiment is short investigation which asks the students to determine the lowest concentration of MB that they can measure. They are asked to calculate this concentration and show the Raman spectrum. In this example, the lowest reliable signal was achieved by adding 2 $\mu \mathrm{l}$ of $1 \times 10^{-6} \mathrm{~mol} \mathrm{dm}^{-3} \mathrm{MB}$ (10-fold dilution of the stock solution supplied) to $2 \mathrm{~cm}^{3}$ of the colloid. Amount of $\mathrm{MB}$ added $=2 \times 10^{-6} \times 1 \times 10^{-6}=2 \times 10^{-12} \mathrm{~mol}$. Volume $=2 \mathrm{~cm}^{3}=2 \times 10^{-3} \mathrm{dm}^{3} . \mathrm{MB}$ Concentration $=2 \times 10^{-12} / 2 \times 10^{-3}=1 \times 10^{-9} \mathrm{~mol} \mathrm{dm}^{-3}$. The Raman Spectrum is shown in Fig. 6 below.

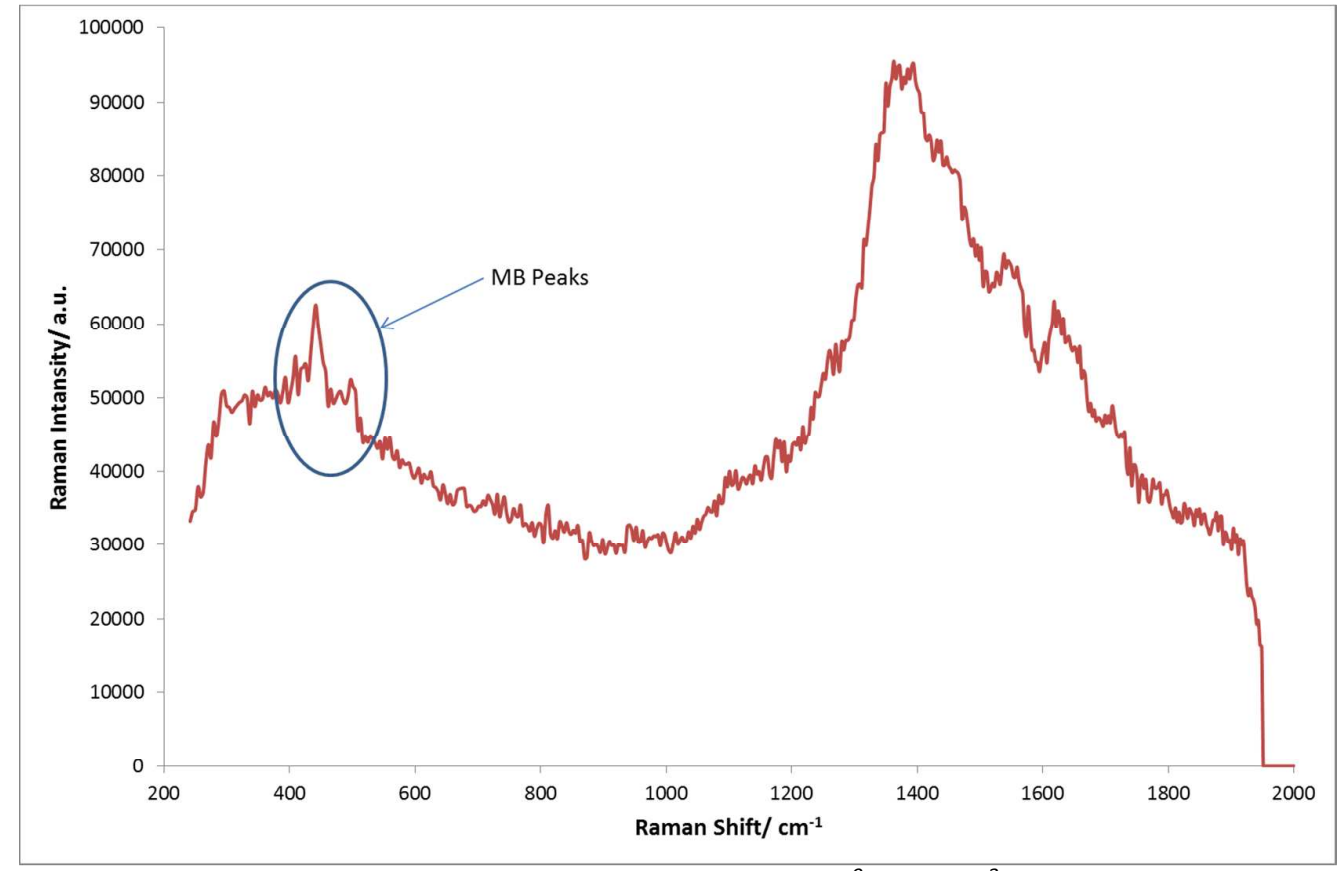

Figure 6-Raman spectrum of $1 \times 10^{-9} \mathrm{~mol} \mathrm{dm}^{-3} \mathrm{MB}$

( 2 marks for chart, 1 mark for reasonable estimate of lowest MB level) 


\title{
DEPARTMENT OF PURE AND APPLIED CHEMISTRY
}

\author{
PHYSICAL AND APPLIED CHEMISTRY \\ $\underline{\text { LABORATORY }}$
}

\section{EXPERIMENT 3C - SURFACE ENHANCED RAMAN SPECTROSCOPY}




\section{RISK ASSESSMENT}

\section{EXPERIMENT 3C - SURFACE ENHANCED RAMAN SPECTROSCOPY}

\begin{tabular}{|c|c|c|c|c|}
\hline Compound & Hazard & Handling & Spillage & Disposal \\
\hline $\begin{array}{c}\text { Sodium } \\
\text { Chloride }\end{array}$ & Irritant & $\begin{array}{c}\text { No special } \\
\text { instructions }\end{array}$ & $\begin{array}{c}\text { Flush with } \\
\text { water }\end{array}$ & $\begin{array}{c}\text { Wash down } \\
\text { sink }\end{array}$ \\
\hline $\begin{array}{c}\text { Sodium } \\
\text { Hydroxide }\end{array}$ & $\begin{array}{c}\text { Corrosive } \\
\text { Irritant }\end{array}$ & $\begin{array}{c}\text { Wear gloves } \\
\text { and wash } \\
\text { hands after } \\
\text { using }\end{array}$ & $\begin{array}{c}\text { Flush with } \\
\text { water }\end{array}$ & $\begin{array}{c}\text { Wash down } \\
\text { sink }\end{array}$ \\
\hline $\begin{array}{c}\text { Silver Nitrate } \\
\text { Hydrochloride }\end{array}$ & $\begin{array}{c}\text { Toxic } \\
\text { Corrosive }\end{array}$ & $\begin{array}{c}\text { Wear gloves } \\
\text { Cormfluse }\end{array}$ & $\begin{array}{c}\text { Flush with } \\
\text { water }\end{array}$ & $\begin{array}{c}\text { Wash down } \\
\text { sink }\end{array}$ \\
\hline $\begin{array}{c}\text { Methylene } \\
\text { Blue }\end{array}$ & Harmful & Wear gloves & $\begin{array}{c}\text { Flush with } \\
\text { water } \\
\text { water }\end{array}$ & $\begin{array}{c}\text { Wash down } \\
\text { sink }\end{array}$ \\
\hline
\end{tabular}




\section{EXPERIMENT 3C}

\section{SURFACE ENHANCED RAMAN SPECTROSCOPY (SERS)}

AIMS

1) To gain an understanding of the potential uses of Raman and UV-VIS spectroscopy.

2) To gain some experience in preparing nanoscale materials.

\section{THEORY}

When a photon interacts with a molecule it can excite it into an electrically polarised virtual energy state. In the vast majority of cases, the excited molecule will rapidly revert to its original energy state and a photon of the same energy is re-emitted/scattered. Occasionally, this excited polarisation state will couple with one of the normal modes of vibration of the molecule. When this happens, the re-emitted photon will have a different energy to the original one and this energy difference will correspond to a vibrational transition: this is known as the Raman Effect. Like IR spectroscopy, Raman Spectroscopy gives information on molecular vibrations. Raman active vibrational modes require a change in molecular polarisability whereas IR active vibrational modes involve a change in dipole moment; this means that IR active modes are not necessarily Raman active and vice versa. These two techniques can therefore offer complementary information on the vibrational modes of molecules.

Historically, the Raman Effect has had limited applicability as only around 1 in $10^{7}$ photons are scattered in this way. Laser technology has changed this, however, as sources of intense, monochromatic light are now readily available.

Recently, substantial research effort has been devoted to nanoscale materials as their properties can be very different from the everyday, large-scale ones. An example is the colloidal suspensions of metal nanoparticles. When metal particles are sufficiently small, the free electrons at the particle's surface can be collectively excited to oscillate by an incoming photon. This collective oscillation is called a surface plasmon, which gives rise to a strong absorption band in the UV-VIS region; these colloidal suspensions are often coloured. The precise frequency of the surface plasmon depends on the size and shape of the metal nanoparticle, so the UV-VIS spectrum of a colloidal suspension will generally show a broad absorption band, with the absorption at each wavelength being proportional to the number of particles having a surface plasmon there. This effect is not observed for large metal particles, as the free electrons cannot be collectively excited by an incoming photon.

If a Raman active molecule is adsorbed on the surface of metal nanoparticle and an incoming photon excites both the surface plasmon and an active vibrational mode then the Raman Effect can be enhanced by a factor of $10^{9}$ times. This effect is called 'Surface Enhanced Raman Spectroscopy' (SERS) and its discovery has allowed single-molecule detection to be achieved. It is important to note that unlike IR spectroscopy, Raman spectroscopy is not a form absorption spectroscopy. Light of a single wavelength is used to excite all of the Raman active vibrational modes, so the Raman scattered, or 'shifted', photons detected will come from all of the allowed vibrational modes. 


\section{EXPERIMENTAL}

\section{$\underline{\text { Synthesis of the Ag Nanoparticle Colloidal Suspension }}$}

The chemical reaction scheme is complex and unresolved, but the hydroxylamine initially reduces the silver ions to form colloidal nanoparticles. The excess hydroxylamine then adsorbs onto the surface of the nanoparticles, both capping their size and preventing aggregation - the capped particles will carry the same electrical charge and hence repel each other. The suspensions remain stable for many months.

In common with many experiments involving nanoscale materials, this experiment involves handling small volumes of dilute solutions. It is very important to avoid unwanted contamination. To help this:

- Do not pipette the stock solutions directly from the bottles - pour the approximate amounts required into small beakers and pipette from these.

- Do not return excess solutions to the bottles, dispose of these down the sink.

- It is recommended that you give all glassware a rinse with deionised water before use. Tap water contains chlorine which will precipitate $\mathbf{A L L}$ of the dissolved $\mathrm{Ag}^{+}$as $\mathrm{AgCl}$ before anything else happens...you don't know how thorough the people doing the experiment before you were at rinsing things, especially in their rush to leave the lab at the end of the day!

- Having said that, please help you fellow students by giving all glassware a final rinse with deionised water before you leave.

1. Pipette $10 \mathrm{~cm}^{3}$ of the $\mathrm{NaOH}$ stock solution into the $100 \mathrm{~cm}^{3}$ volumetric flask.

2. Using the glass weighing boat provided, weigh out $(0.100 \pm 0.005) \mathrm{g}$ of hydroxylamine hydrochloride. WEAR GLOVES.

3. Carefully rinse the hydroxylamine directly into the $\mathrm{NaOH}$ using deionised water and make the solution up to the mark. Invert the flask several times to fully dissolve the salt.

4. Pipette $50 \mathrm{~cm}^{3}$ of the $\mathrm{AgNO}_{3}$ stock solution into a $100 \mathrm{~cm}^{3}$ conical flask, add a magnetic stirrer bar and set the stirring rate to be slow and steady.

5. Pipette $5 \mathrm{~cm}^{3}$ of your Hydroxylamine/ $\mathrm{NaOH}$ solution into the $\mathrm{AgNO}_{3}$ and continue the stirring for the rest of today's experiment. The colour should initially change to grey and then become milky yellow/brown - ask if unsure, the success of the experiment depends on the colloid. 


\section{UV-VIS}

1. Open the SpectraSuite software from the desktop: you should see the screen shown in Fig 1.

2. Close the 'Graph (A)' tab as shown.

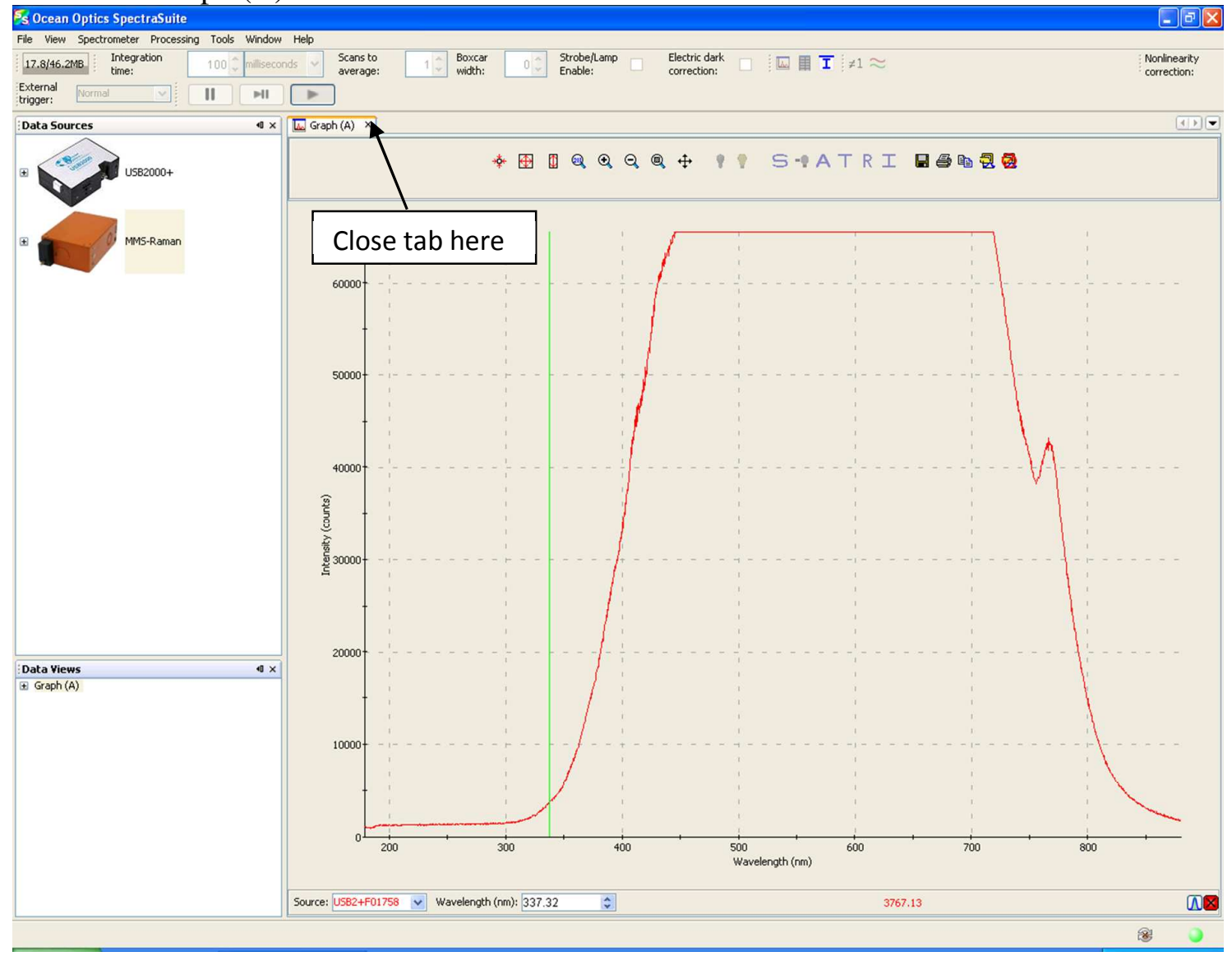

Figure 1 - SpectraSuite Screen Shot

3. From the 'File' menu select 'New Absorbance Measurement'.

4. Ensure that the USB2000+ instrument is highlighted then click 'Next'.

5. Set 'Integration time' to $10 \mathrm{~ms}$, 'Scans to average' to 20, 'Boxcar width' to 20 and click 'Next'.

6. Fill the cuvette to the black line with deionised water, place it into the cell holder, press the yellow light bulb icon to record the baseline spectrum and click 'Next'.

7. Remove the cuvette and replace it with the darkened one. Press the grey light bulb icon to measure a dark spectrum and then click 'Finish'.

8. Press the button and under X-Axis Range set Minimum to $350 \mathrm{~nm}$ and Maximunı w $820 \mathrm{~nm}$. Under the Y-Axis Range set Minimum to 0.0 and Maximum to 1.8. Click 'Apply' then 'Close'.

9. Attach a new $1 \mathrm{~cm}^{3}$ tip to the large micropipette and ensure that the pipette is set to read ' 100 ', this sets the pipette to deliver $1 \mathrm{~cm}^{3}$. Pipette $1 \mathrm{~cm}^{3}$ of your prepared Ag colloid into the cuvette of deionised water. To do this, slowly depress the plunger down until it first stops, insert the tip into the suspension and slowly raise the plunger to fill the tip. Discharge the suspension into the water by FULLY depressing the plunger. Invert several times to mix.

10. Place the cuvette into the spectrometer; the absorbance spectrum should appear. 
11. Click 蹋 to copy the data. Open Excel and paste the data into a spreadsheet under the heading UV-VIS NO NaCl.

12. Add 2 drops of $\mathrm{NaCl}$ solution to the cuvette, mix and replace in the spectrometer. Wait for 1 minute and copy and paste these data into your spreadsheet under the heading UV-VIS AND NaCl.

13. Save your spreadsheet, remove and rinse the cuvette and switch off the UV-VIS lamp.

14. Close the 'Graph (B)' tab on the SpectraSuite window.

\section{$\underline{\text { Raman: Part A - Concentration Dependence }}$}

1. Turn on the MMS-RAMAN Spectrometer by turning the key on the front clockwise. Make sure the red light on the front comes on (see Fig. 2).

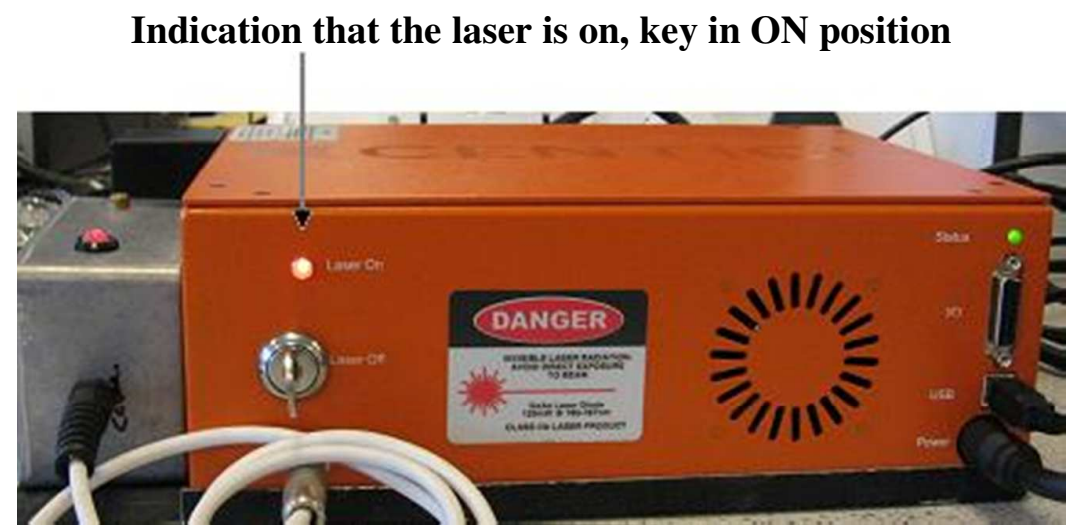

Figure 2 - Photograph of MMS-RAMAN Spectrometer

2. Open Excel and set up a spreadsheet with the following headings:

\begin{tabular}{|c|c|c|c|c|c|c|c|c|c|c|c|c|c|c|}
\hline 4 & A & B & C & D & $E$ & $\mathrm{~F}$ & G & H & 1 & J & $\mathrm{K}$ & L & M & $\mathrm{N}$ \\
\hline 1 & Colloid & & & MB & & & $10 \mu \mathrm{l} M B$ & & & $10 \mu \mathrm{MB}+\mathrm{NaCl}$ & & & Most Dilute Spectrum & \\
\hline 2 & Raman Shift $/ \mathrm{cm}^{-1}$ & Intensity/a.u. & & Raman Shift $/ \mathrm{cm}^{-1}$ & Intensity/a.u. & & Raman Shift $/ \mathrm{cm}^{-1}$ & Intensity/a.u. & & Raman Shift $/ \mathrm{cm}^{-1}$ & Intensity/a.u. & & Raman Shift $/ \mathrm{cm}^{-1}$ & Intensity/a.u. \\
\hline 3 & & & & & & & & & & & & & & \\
\hline 4 & & & & & & & & & & & & & & \\
\hline
\end{tabular}

Figure 3 - Excel Spreadsheet

3. In SpectraSuite, click the picture of the MMS-Raman instrument to highlight it as shown in Fig 4.

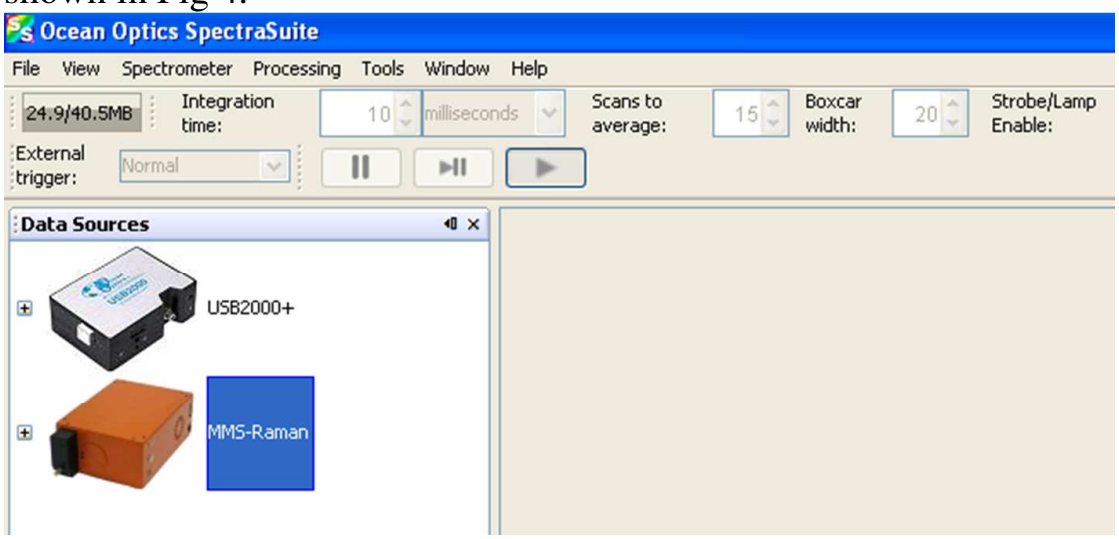

Figure 4 - Screen Shot

4. From the 'File' menu, select 'New Spectrum Graph'. Set the Integration time to $5 \mathrm{~s}$ (select 'seconds' from the dropdown menu before setting the number '5'). See Fig. 5. 


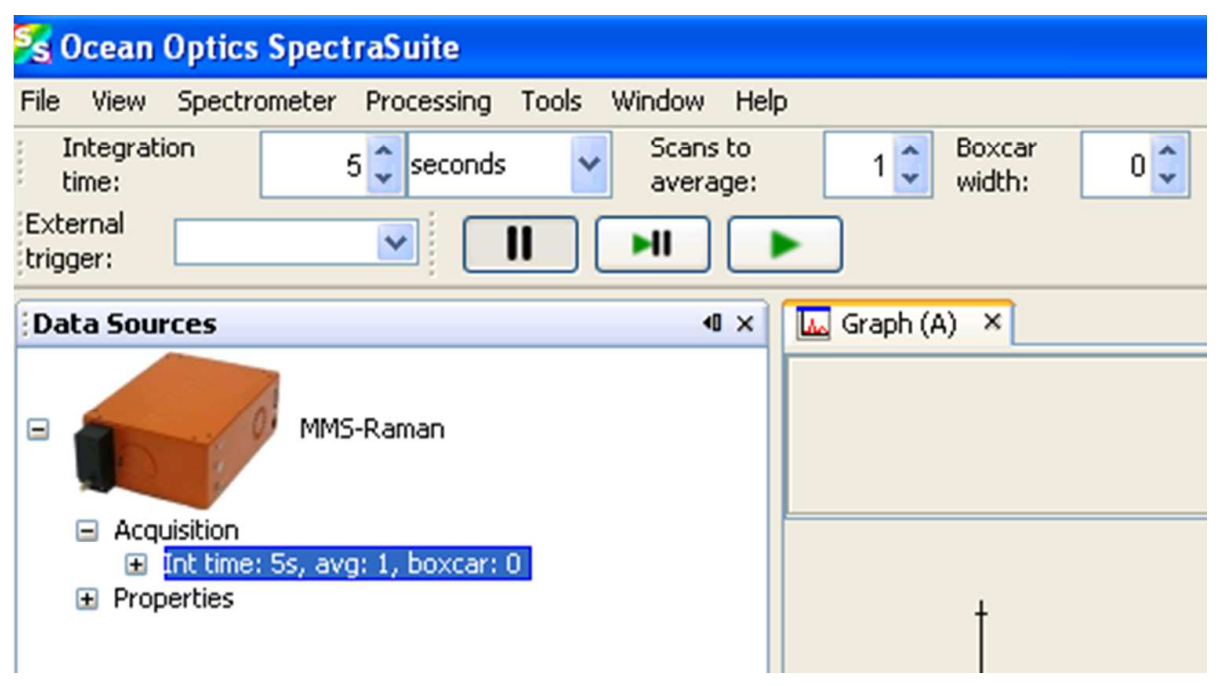

Figure 5 - SpectraSuite Interface

5. From the 'Processing' menu, select 'Processing Mode' then 'Raman Shifts'.

6. Pipette $2 \mathrm{~cm}^{3}$ of your colloid into each of two $2 \mathrm{~cm}^{3}$ glass vials. Place one aside for use in Part $\mathrm{C}$.

7. Lift off the black metal cover on the spectrometer. This will automatically turn off the laser (the red light on top turns green). Place your sample vial into the sample bay and replace the cover. The light on top should now again be red, indicating that the laser is on. This is all illustrated in Fig. 6.
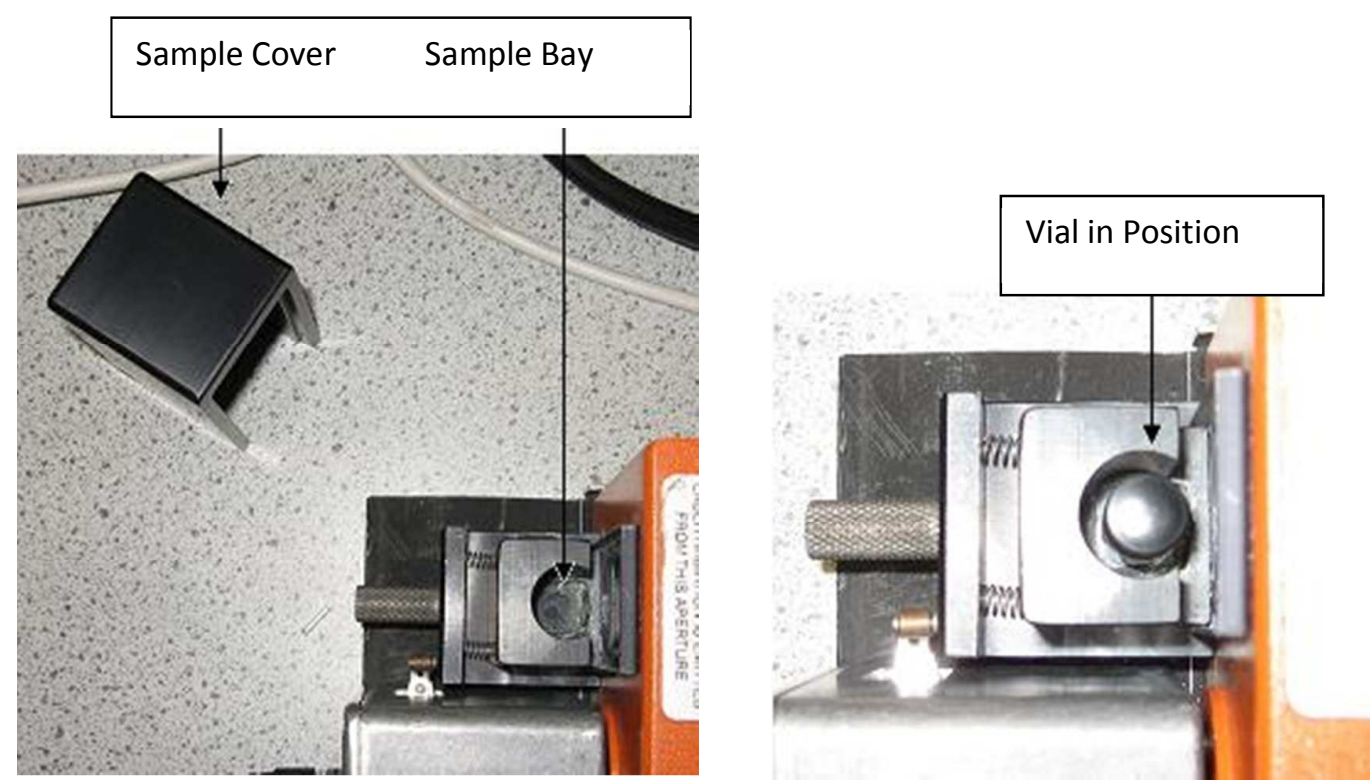

Figure 6 - Sample Placement

8. Click the $\boxplus$ button next to the picture of the Spectrometer on the left of the screen to expand the menu. Now click the $\boxplus$ next to 'Acquisition'. Click to highlight the line that says 'Int time: 5s, avg: 1, boxcar: 0'. It should turn blue as in Fig. 5.

9. Measure the spectrum by clicking the $\quad$ II button. 
10. Once the spectrum has been measured, click the button to resize it to full screen and then click 筩 to copy the data to the clipboard. Bring up Excel and paste the data into the spreadsheet. Save your spreadsheet now and after every change you make.

11. Retain your vial of Ag colloid for later.

12. Using a clean, glass Pasteur pipette, fill a new vial with the $1 \times 10^{-4} \mathrm{M}$ methylene blue stock solution and measure its Raman spectrum. You will have to click to highlight the 'Int time: 5s, avg: 1, boxcar: 0' line every time you want to run a spectrum.

13. Copy and paste these data into your Excel spreadsheet.

14. Place a new tip onto the smaller micropipette and set this to read ' 020 '. This delivers $2 \mu \mathrm{l}\left(2.0 \times 10^{-3} \mathrm{~cm}^{3}\right)$ of solution.

15. Pipette $2 \mu \mathrm{l}$ of the methylene blue stock solution into your vial of Ag colloid and invert several times to thoroughly mix. (NOTE: When pipetting $2 \mu$, the first stop of the pipette plunger will occur very quickly, it is therefore important to do this slowly. Consult a demonstrator if you are unsure.)

16. Measure the Raman spectrum of your sample, clickin: $\mathrm{O}$ n to resize your spectrum. Do not copy and paste the spectrum at this stage.

17. Click on the spectrum and then click on the integration button $\mathbb{N}$ at the bottom right of the screen.

18. Double click first main peak at $c a .430 \mathrm{~nm}$ to move the green cursor there and then use $\lambda \triangle B$ buttons to position the cursor exactly on the peak. Read off the Raman Intensity as shown in Fig. 7 and write this in Table 1.

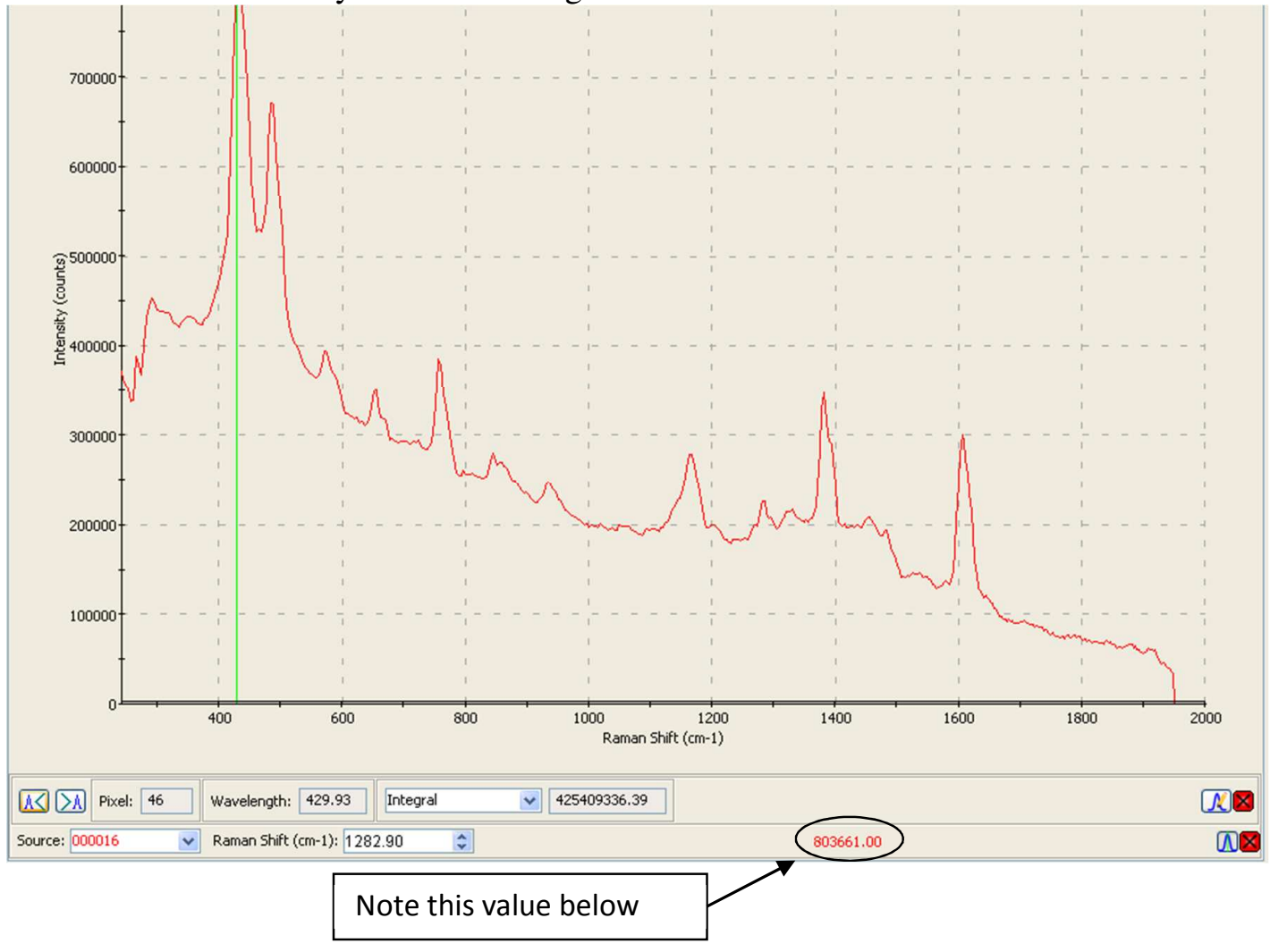

Figure 7 - Raman Peak Evaluation 


\begin{tabular}{|c|c|c|}
\hline Volume Added $/ \mu \mathrm{l}$ & Concentration/ $\mathrm{mol} \mathrm{dm}^{-3}$ & Raman Intensity/ a.u. \\
\hline 2.0 & $1.0 \times 10^{-7}$ & \\
\hline 4.0 & $2.0 \times 10^{-7}$ & \\
\hline 6.0 & $3.0 \times 10^{-7}$ & \\
\hline 8.0 & $4.0 \times 10^{-7}$ & \\
\hline 10.0 & $5.0 \times 10^{-7}$ & \\
\hline
\end{tabular}

Table 1 - Raman Intensity \& Methylene Blue Concentration

19. Repeat steps 15 - 18 a further 4 times until you have added a total of $10 \mu \mathrm{l}$ of methylene blue to your sample. Only copy and paste your $10 \mu \mathrm{l}$ sample data into Excel.

20. Add one drop of $\mathrm{NaCl}$ solution to the vial, mix well and re-measure the Raman Spectrum after $\sim 1$ minute. Resize, and copy and paste your data. $\mathrm{NaCl}$ causes aggregation of the colloidal particles.

\section{$\underline{\text { Raman: Part B - Integration Time Dependence }}$}

1. Pipette $2 \mathrm{~cm}^{3}$ of your colloid into a new sample vial and add $4 \mu \mathrm{l}$ of methylene blue.

2. Change the Integration time to $1 \mathrm{~s}$ and record the Raman Spectrum as before, noting the integration value of the large peak in the table below. There is no need to copy and paste your spectral data.

3. Complete Table 2 for the given integration times. Do not remove the sample from the spectrometer, just change the integration time.

\begin{tabular}{|c|c|}
\hline Integration Time/ s & Raman Intensity/ a.u. \\
\hline 1 & \\
\hline 2 & \\
\hline 5 & \\
\hline 8 & \\
\hline 10 & \\
\hline 12 & \\
\hline 15 & \\
\hline 18 & \\
\hline 20 & \\
\hline
\end{tabular}

Table 2 - Raman Intensity \& Integration Time

\section{$\underline{\text { Raman: Part } \mathrm{C} \text { - Investigation }}$}

What is the lowest methylene blue concentration that you can reliably measure using this technique? Here is a guide on how to proceed.

- Dilute the methylene blue stock solution by e.g. pipetting 0.5 or $1 \mathrm{~cm}^{3}$ into a $100 \mathrm{~cm}^{3}$ volumetric flask and making up to the mark.

- Place the vial of colloid that you set aside in Part A into the spectrometer, select your integration time and record a blank spectrum (if it's not blank, consult a demonstrator).

- Add $1 \mu \mathrm{l}$ of your diluted methylene blue solution to the colloid. Do you see a definite methylene blue signal? Add further $1 \mu \mathrm{l}$ aliquots and measure a spectrum after each 
addition until you see the first definite signal. What else could you add to the colloid to confirm that you were measuring the SERS spectrum? With this in mind, could you improve the detection level further?

- Copy and paste your most dilute, yet positive, response into your spreadsheet and explain what you did in your report.

At the end of the experiment, rinse all glassware and cells with deionised water-please help us to avoid contaminating the glassware (particularly with $\mathrm{NaCl}$ ) for the next students.

\section{DATA RETRIEVAL}

You should have your UV-VIS and Raman data on spreadsheets. In your UV-VIS data, the first column is $\lambda(\mathrm{nm})$ and the second is absorbance. Each Raman spectrum has two columns of data. The first column is the Raman Shift $\left(\mathrm{cm}^{-1}\right)$ and the next is the Raman Intensity (a.u.). Your spectra will simply be plots of Raman Intensity vs. Raman Shift.

\section{QUESTIONS AND ANALYSIS}

Q1 On the same chart, plot your UV-VIS spectra for the diluted Ag colloid both with and without added $\mathrm{NaCl}$. You should plot absorbance vs. wavelength in the $350 \mathrm{~nm}$ $820 \mathrm{~nm}$ range only.

Q2 On a new chart, plot the Raman spectra of both the Ag colloid and the methylene blue stock solution. These are the first two spectra that you recorded. Indicate some of the (weak) methylene blue Raman bands on your spectrum.

Q3 On a new chart, plot the Raman spectra of the methylene blue stock solution, the 10 $\mu \mathrm{l}$ methylene blue/Ag colloid and the $10 \mu \mathrm{l}$ methylene blue/Ag colloid/ $\mathrm{NaCl}$. Comment on the differences between the three spectra.

Q4 Given that the laser wavelength is $784 \mathrm{~nm}$, explain the difference in the Raman scattering intensity when the $\mathrm{NaCl}$ is added. You should refer to your UV-VIS spectra.

Q5 Include Table 1 in your report and plot Raman Intensity vs. Concentration - comment on these data.

Q6 Include Table 2 in your report and plot Raman Intensity vs. Integration Time comment on these data.

Q7 What is the lowest methylene blue concentration that you were able to detect (in mol $\left.\mathrm{dm}^{-3}\right)$ ? Remember that the volume of colloid added was $2 \mathrm{~cm}^{3}$ and this needs to be accounted for when calculating your final concentration. Show your calculation and your Raman spectrum. 


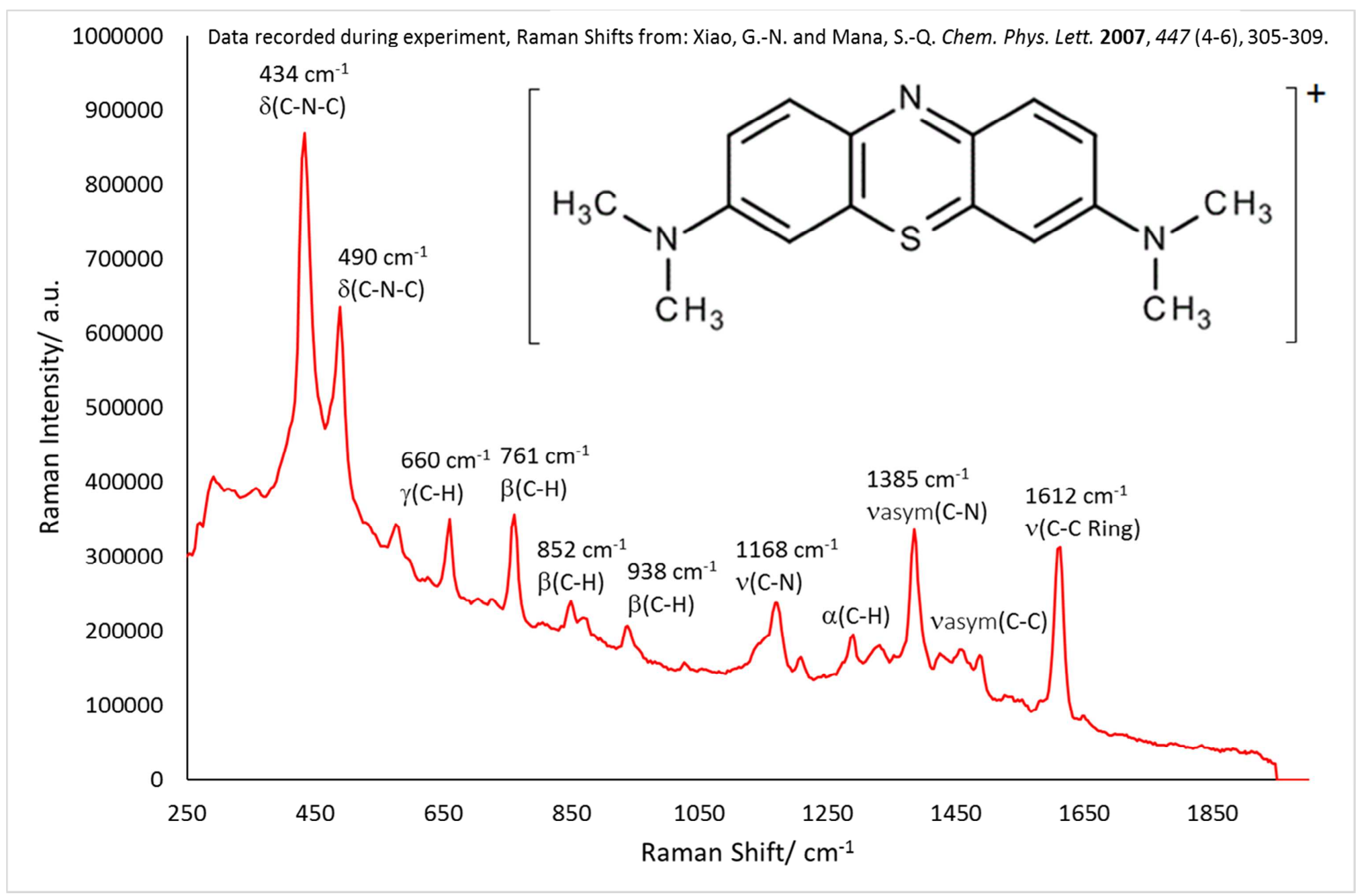

